\title{
Fast Chaos with Slow $p-n$ Junction Diodes
}

\author{
G. Mykolaitis ${ }^{a, b, *}$, R. MiŠKinis $^{a}$, S. BumelienE ${ }^{a}$, \\ E. TAMAŠEVIČIŪtét $\dot{E}^{a, c}$ AND A. TAMAŠEVIČIUSS ${ }^{a}$ \\ ${ }^{a}$ Semiconductor Physics Institute \\ A. Goštauto 11, LT- 01108 Vilnius, Lithuania \\ ${ }^{b}$ Department of Physics, Vilnius Gediminas Technical University \\ Saulètekio Av. 11, LT-10223 Vilnius, Lithuania \\ ${ }^{c}$ Department of Radiophysics, Vilnius University \\ Saulètekio Av. 9, LT-10222 Vilnius, Lithuania
}

\begin{abstract}
We demonstrate both experimentally and numerically that slow recovery $p-n$ junction diodes can be exploited to generate chaos at high megahertz frequencies. An extremely simple resonator consisting of an inductor in parallel with a diode when externally periodically driven exhibits chaotic response.
\end{abstract}

PACS numbers: 05.45.Ac, 05.45.Pq, 05.45.Gg, 85.30.Kk

\section{Introduction}

Three years ago at the 12-UFPS symposium we suggested high-speed Schottky barrier diodes to generate chaotic wave forms in very high frequency (30 to $300 \mathrm{MHz}$ ) band. Fast recovery $(25 \mathrm{ps})$ and low barrier capacitance $(0.3 \mathrm{pF}) \mathrm{mi}-$ crowave diodes exhibited upper spectral limit of $250 \mathrm{MHz}$ [1]. In the present paper, in contrast to the previous work, we propose to use slow recovery and moderate capacitance $p-n$ junction diodes in chaotic oscillators. Diode resonator is known for many years [2-9] and has been extensively investigated theoretically and experimentally. There was a long lasting controversy about the physical mechanism behind the chaotic response of the diode, e.g. in the early comments [4, 5] and in a lot of subsequent works. Experiments have been carried out at relatively low frequencies from $50 \mathrm{kHz}$ to $10 \mathrm{MHz}$ [2-4, 7-9]. However, for many applications chaotic wave forms of hundreds of megahertz are required.

\section{Experimental setup}

The diode resonator (D-resonator) is commonly a series circuit comprising a resistor $R$, an inductor $L$, and a $p-n$ junction diode $D$ [2-9]. When periodically

\footnotetext{
*corresponding author; e-mail: gytis@pfi.lt
} 
driven by external voltage source $V_{0} \sin \omega t$ (Fig. 1a) it exhibits chaotic response at a certain set of control parameters $V_{0}, \omega, R$, and $L$. Important conditions
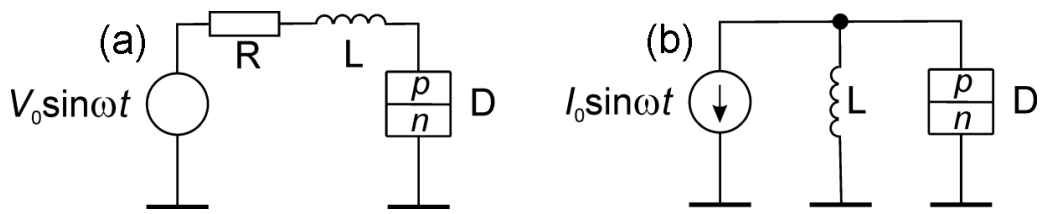

Fig. 1. Diode resonators. (a) Series coupling to voltage source. (b) Parallel coupling to current source.

are $\omega \approx \omega_{\text {res }}=\left(L C_{\mathrm{j}}\right)^{-1 / 2}$ and $0<R<0.1\left(L / C_{\mathrm{j}}\right)^{1 / 2}$, where $C_{\mathrm{j}}$ is the junction capacitance. The $\omega_{\text {res }}$ can be increased by decreasing either both or one of the values, $L$ and/or $C_{\mathrm{j}}$. Tuning of $L$ is a more convenient way, since it is an external circuit parameter, while $C_{\mathrm{j}}$ is an intrinsic characteristic of the diode. However, with the decrease in $L$ it becomes difficult to satisfy $R<0.1\left(L / C_{\mathrm{j}}\right)^{1 / 2}$, because the output resistance $R_{\text {out }}=50 \Omega$ of a real periodic oscillator starts to play the role of $R$. Therefore, at higher frequencies we suggest a parallel configuration of the D-resonator with a periodic current source $I_{0} \sin \omega t$ (Fig. 1b).

\section{Experimental results}

Here we present experimental evidence of chaotic response of the D-resonator with silicon $p-n$ junction diodes characterized by large recovery time $\left(\tau_{\text {rec }} \approx 1 \mu \mathrm{s}\right)$ and moderate junction capacitance $\left(C_{\mathrm{j}}=20 \mathrm{pF}\right)$ in high frequency (3 to $\left.30 \mathrm{MHz}\right)$ band. Chaotic nature of the response is confirmed by continuous and broadband character of the power spectrum (Fig. 2a). In contrast to many other

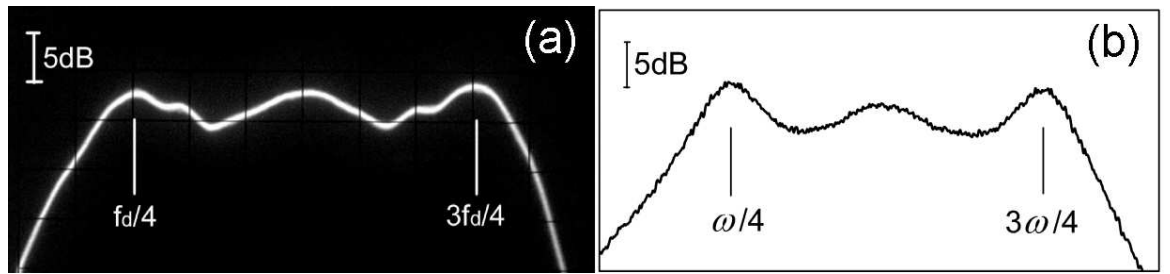

Fig. 2. Power spectra. (a) Experimental, $f_{\mathrm{d}}=42 \mathrm{MHz}, L=1 \mu \mathrm{H}$. (b) Fourier transform of $x(t)$ in Eq. (4.2) with $a=5, b=20, c=500, \omega=0.8$.

chaotic oscillators, including series type D-resonators, power spectrum of the parallel D-resonator is extremely broad-band and relatively flat $(\approx 5 \mathrm{~dB})$. It occupies frequency band between some definite sub-harmonic limits, e.g. $f_{\mathrm{d}} / 4$ and $3 \mathrm{fd} / 4$ and has sharp falls outside them. The fractional spectral band width $\Delta=2\left(f_{\mathrm{H}}-f_{\mathrm{L}}\right) /\left(f_{\mathrm{H}}+f_{\mathrm{L}}\right)=1$. It satisfies the criteria $(\Delta>0.25)$ for the ultrawide-band signals. In addition, ultrahigh frequency $(300 \mathrm{MHz}$ to $1 \mathrm{GHz})$, also 
$L$ band (1 to $2 \mathrm{GHz}$ ) experiments are in progress and show qualitatively similar results.

\section{Mathematical model}

The D-resonator in Fig. 1b is described by the following equations:

$$
\dot{Q}=-I_{L}+I_{0} \sin \omega t-I(V), \quad \dot{I}_{L}=V .
$$

The $Q(V)=C_{\mathrm{j}}(V) V+\tau_{p} I(V)$ is the total charge in the $p-n$ junction, $\tau_{p}$ is the lifetime of the excess carriers, $V$ is the voltage across the diode, $I_{L}$ and $I(V)$ is the current through the inductor and the diode, respectively. Equations (4.1) can be represented in a more convenient dimensionless form

$$
h(x) \dot{x}=-y+a \sin \omega t-b g(x), \quad \dot{y}=x .
$$

Here $x \propto V, y \propto I_{L}$. The nonlinear functions $g(x)$ and $h(x)$ are given by

$$
\begin{aligned}
& g(x)=(x-1) H(x-1), \quad h(x)=1+c H(x-1), \\
& a=I_{0} \sqrt{L / C_{\mathrm{j}}} / V^{*}, \quad b=\sqrt{L / C_{\mathrm{j}}} / R_{\mathrm{d}}, \\
& c=C_{\text {diff }} / C_{\mathrm{j}}=\tau_{p} / R_{\mathrm{d}} C_{\mathrm{j}} .
\end{aligned}
$$

$H(\cdot)$ is the Heaviside function. The $V^{*}, R_{\mathrm{d}}, C_{\mathrm{diff}}=\tau_{p} / R_{\mathrm{d}}$, and $C_{\mathrm{j}}$ are forward voltage drop, the differential resistance, diffusive, and junction capacitance of the diode, respectively. In $h(x)$ weak dependence of the $C_{\mathrm{j}}$ on $\mathrm{V}$ is neglected.

\section{Numerical results}

Equations (4.2) have been integrated numerically and the results are presented in Fig. 2b and Fig. 3. Time series exhibits "random" voltage spikes across the closed diode. Chaos is observed only at high values of the control parameter $c$, e.g. at $c>50$ for reasonable values of the other coefficients.

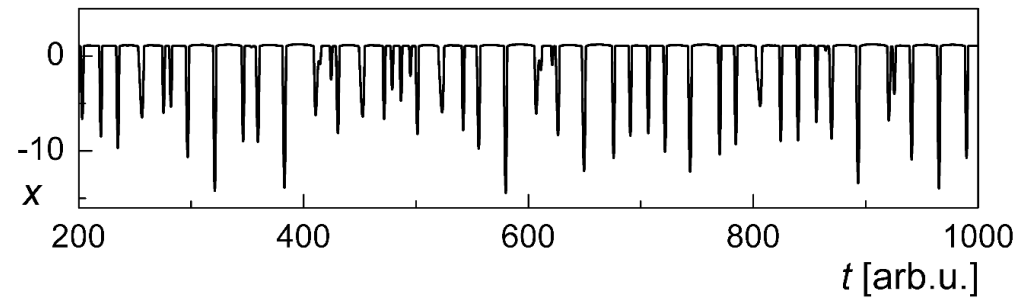

Fig. 3. Time series $x(t)$ from Eq. (4.2) with $a=5, b=20, c=500, \omega=0.8$. 


\section{Conclusions}

Slow recovery $p-n$ junction diodes can be successfully employed in externally driven parallel diode resonator to generate high frequency chaotic wave forms. Chaotic response of the diode resonator is conditioned by high ratio of the diffusive capacitance $C_{\mathrm{diff}}$ to the junction capacitance $C_{\mathrm{j}}$ of the diode.

\section{Acknowledgments}

E.T. was partly supported by the Gifted Student Fund-Lithuania.

\section{References}

[1] G. Mykolaitis, A. Tamaševičis, S. Bumelienè, K. Pyragas, V. Pyragas, Acta Phys. Polon. A 107, 365 (2005).

[2] P.S. Linsay, Phys. Rev. Lett. 47, 1349 (1981).

[3] J. Testa, J. Perez, C. Jeffries, Phys. Rev. Lett. 48, 714 (1982).

[4] E. Hunt, Phys. Rev. Lett. 49, 1054 (1982).

[5] J. Testa, J. Perez, C. Jeffries, Phys. Rev. Lett. 49, 1055 (1982).

[6] R.W. Rollins, E. Hunt, Phys. Rev. Lett. 49, 1295 (1982).

[7] T. Matsumoto, L.O. Chua, S. Tanaka, Phys. Rev. A 30, 1155 (1984).

[8] D.J. Gauthier, D.W. Sukow, H.M. Concannon, J.E.S. Socolar, Phys. Rev. E 50, 2343 (1994).

[9] S. Tanaka, S. Higuchi, T. Matsumoto, Phys. Rev. E 54, 6014 (1996). 